\title{
FIVE OPEN PROBLEMS IN COMPRESSIBLE MATHEMATICAL FLUID DYNAMICS*
}

\author{
DENIS SERRE ${ }^{\dagger}$
}

\begin{abstract}
The problems below are motivated by some of the works I did in the past. They deal with the dynamics of compressible fluids, either viscous or inviscid. They could be good questions for mathematicians having originality and technical strength. They are not worth a million dollars, even not a penny, but they are interesting in their own.
\end{abstract}

Key words. Euler equations, shock waves, vacuum, compensated compactness.

AMS subject classifications. 76N10, 76N15, 76L05, 35L60, 35L65, 35L67.

\section{Global-in-time Cauchy problem for Euler-Fourier system.}

Problem \#1. To develop a global-in-time theory of the Cauchy problem for the 1-D Euler-Fourier system. Initial data would only be constrained by finite energy and entropy, and possibly by local boundedness of appropriate quantities, like $\rho, \frac{1}{\rho}, v, \theta, \frac{1}{\theta}$.

The complete Navier-Stokes-Fourier system for a viscous, heat-conducting fluid is

$$
\begin{aligned}
\partial_{t} \rho+\operatorname{div}(\rho v) & =0 \\
\partial_{t}(\rho v)+\operatorname{Div}(\rho v \otimes v)+\nabla p(\rho, e) & =\operatorname{Div} \mathcal{T} \\
\partial_{t}\left(\frac{1}{2} \rho|v|^{2}+\rho e\right)+\operatorname{div}\left(\frac{1}{2} \rho|v|^{2}+\rho e+p(\rho, e)\right) v & =\operatorname{div}(\mathcal{T} v+\kappa(\rho, e) \nabla \theta),
\end{aligned}
$$

where the viscous tensor is given by

$$
\mathcal{T}:=\mu(\rho, e)\left(\nabla v+(\nabla v)^{T}\right)+(\zeta(\rho, e)-2 \mu(\rho, e))(\operatorname{div} v) I_{d}
$$

Under appropriate assumptions about the pressure law $(\rho, e) \mapsto p$ and the diffusion coefficients $\mu, \zeta$ and $\kappa$, the existence of global-in-time renormalized solutions to the Cauchy problem has been proved for finite energy/entropy initial data $[1,9,10,12]$. Local-in-time existence of smooth solutions is also known when the initial data is smooth enough. By smooth, we mean data of class $H^{s}$ with $s>1+\frac{d}{2}, d$ the space dimension; the corresponding solutions are almost classical ones. See [15, 16, 26].

One may wonder what happens if one of the diffusion processes is negligible. We explained in [25] that the only meaningful limit is that towards the Euler-Fourier system, for which $\mu, \zeta \equiv 0$ but $\kappa>0$. In particular, we proved that for a smooth initial data, the local-in-time smooth solution provided by S. Kawashima's theory converges as $\mu, \zeta \rightarrow 0+$ towards the local-in-time smooth solution of the Euler-Fourier system.

When the initial data is not smooth, but has finite energy and entropy, the Cauchy problem for the Euler-Fourier system seems to be an open problem; even in one space dimension!

Why is it difficult? Let us first recall some basic facts from [15, 26].

\footnotetext{
*Received September 7, 2012; accepted for publication January 25, 2013.

†UMPA, UMR CNRS-ENS Lyon \# 5669, École Normale Supérieure de Lyon, 46, allée d'Italie, F-69364 Lyon, cedex 07, France (denis.serre@ens-lyon.fr).
} 
Viscous system of conservation laws have the general form

$$
\partial_{t} u+\operatorname{Div} F(u)=\operatorname{Div}(B(u) \nabla u):=\sum_{\alpha, \beta} \partial_{\alpha}\left(B^{\alpha \beta}(u) \partial_{\beta} u\right),
$$

where $u(x, t) \in \mathbb{R}^{n}$ is the vector-valued unknown. Basic assumptions are

- The system (1) dissipates a strongly convex entropy $\eta(u)$,

- The $p$ first lines of the system are first-order; with $w=\left(u_{1}, \ldots, u_{p}\right)$, they read

$$
\partial_{t} w+\operatorname{Div} f(u)=0
$$

- The rank of the symbol

$$
B(u ; \xi)=\sum_{\alpha, \beta} \xi_{\alpha} \xi_{\beta} B^{\alpha \beta}(u)
$$

equals $n-p$ for every $\xi \neq 0$. Its range is therefore constant, equal to $\{0\} \times$ $\mathbb{R}^{n-p}$.

We proved in [26] the following facts

- The dissipation tensor $B(u) \nabla u$ involves only the gradients $\nabla z_{j}$ for $j=p+$ $1, \ldots, n$ where

$$
z_{j}=\frac{\partial \eta}{\partial u_{j}}
$$

Defining $z:=\left(z_{p+1}, \ldots, z_{n}\right)$, the first-order operator $z \mapsto B(u) \nabla u=$ $\left(\begin{array}{c}0 \\ b(u) \nabla z\end{array}\right)$ is elliptic.

- Let $g$ be defined from $f$ by the change of variable $u \mapsto(w, z)$, that is $g(w, z)=$ $f(u)$. When $z$ is frozen, the system

$$
\partial_{t} w+\operatorname{Div} g(w, z)=0 .
$$

is hyperbolic. It is called the reduced inviscid system. Actually, it admits a strongly convex entropy $E$ which is evaluated from $\eta$ after two Legendre transforms $\eta \mapsto \eta^{*}$ (with respect to the variable $u$ ) and $\eta^{*} \mapsto E$ (with respect to $z_{1}, \ldots, z_{p}$, while $z_{p+1}, \ldots, z_{n}$ are frozen).

It turns out that the system (1) displays both parabolic features (according to the variable $z$ ) and hyperbolic ones (according to $w$ ). The hyperbolic waves are convected at the velocities of system (2).

The reduced system (2) plays another important role in the analysis of (1). For instance, it governs the jump relations (Rankine-Hugoniot relations) in discontinuous solutions. It can be genuinely nonlinear or linearly degenerate. In the former case, the solutions of (2) (at constant $z$ ) usually devellop shock waves in finite time. In the full system (1), this phenomenon remains possible: $w$ becomes discontinuous in finite time, while $z$ does not, because of the parabolic dissipation. However, $\nabla z$ becomes discontinuous when $w$ does, due to the coupling. Actually, there is a competition between the damping induced by dissipation plus coupling on the one hand, and the hyperbolic non-linearity on the other hand. Therefore a small smooth initial data yield a global-in-time solution, provided that the coupling is strong enough, in the sense that the eigenvectors of the hyperbolic symbol

$$
A(u ; \xi)=\sum_{\alpha} \xi_{\alpha} \mathrm{D} F^{\alpha}(u)
$$


do not belong to the kernel of $B(u ; \xi)$. This property bears the name of KawashimaShizuta condition.

The possibility that $w$ becomes discontinuous renders the analysis of the Cauchy problem especially difficult. Morally, we don't expect to solve the full Cauchy problem before being able to solve that for the reduced system (2); somehow, all the difficulties associated with (2) are present in (1).

Of course, when (2) is linearly degenerate, the situation is much nicer, because we expect that the regularity of $v$ be propagated.

Back to Gas Dynamics. We distinguish two cases, depending on whether we take in account both viscosity and heat conduction, or only heat conduction:

- In the Navier-Stokes-Fourier system, we have $p=1, w=\rho$ and $z=\left(\frac{v}{\theta},-\frac{1}{\theta}\right)$. The reduced equation

$$
\partial_{t} \rho+\operatorname{div}(\rho v)=0
$$

is a transport equation with constant velocity (here, $v$ is frozen because the viscosity does involve $\nabla v$ ). Not only (2) is linearly degenerate, but it is even linear. This is a very nice situation; for instance the discontinuities of $\rho$ may propagate, and they actually do, but they may not appear from nowhere. This explains why this case has been so far the only one for which the global existence was proved for the Cauchy problem with large initial data.

- In the Euler-Fourier system, there is only heat conduction. Then $p=1+d$ and $w=(\rho, \rho v), z=-\frac{1}{\theta}$. The reduced system is the Euler system for a isothermal gas; it is genuinely non-linear. The theory of the quasi-linear hyperbolic Cauchy problem for large initial data is still lacking if $d \geq 2$, so we don't expect any immediate progress for the multi-dimensional Euler-Fourier system.

This leaves open the one-dimensional case

$$
\begin{aligned}
\partial_{t} \rho+\partial_{x}(\rho v) & =0 \\
\partial_{t}(\rho v)+\partial_{x}\left(\rho v^{2}+p(\rho, e)\right) & =0 \\
\partial_{t}\left(\frac{1}{2} \rho v^{2}+\rho e\right)+\partial_{x}\left(\frac{1}{2} \rho v^{2}+\rho e+p\right) v & =\partial_{x}\left(\kappa \partial_{x} \theta\right) .
\end{aligned}
$$

The reduced system is a $2 \times 2$ hyperbolic system

$$
\begin{aligned}
\partial_{t} \rho+\partial_{x}(\rho v) & =0 \\
\partial_{t}(\rho v)+\partial_{x}\left(\rho v^{2}+p(\rho)\right) & =0 .
\end{aligned}
$$

The hyperbolic Cauchy problem with large data can be attacked by the compensated compactness method, as done by R. DiPerna [7] and followers (G.Q. Chen \& coll. [8], P.-L. Lions, B. Perthame, P. Souganidis \& E. Tadmor $[20,19]$, Ph. LeFloch [17]). It is not clear how compensated compactness could be adapted to a partially parabolic context. A seamingly better situation arises if we think that for a perfect gas, the isothermal motions obey to the pressure law $p=A^{2} \rho$, where $A=A(\theta)$. Then the reduced system is the one analyzed by $\mathrm{T}$. Nishida [22]; it has the property that the density stays away from zero if it is positive at initial time. In addition, there is a Lyapunov function, analogous to the total variation of the Riemann invariants, which makes the Glimm scheme particularly tractable. But again, it is unclear how 
to incorporate the Glimm scheme, even in its simplest form à la Nishida, into an analysis of a coupled hyperbolic-parabolic system.

We point out one difficulty that is common to all mathematical questions about compressible gases: it is unclear a priori whether the density remains positive everywhere. The vacuum renders the equations singular in some sense; at least, the first-order part (convection terms) looses hyperbolicity. Even in one space-dimension, this difficulty necessitates a specific treatment. See the works by compensated compactness, for instance $[19,20,5]$.

\section{2D compressible Euler system.}

Problem \#2. To develop a Compensated Compactness calculus about symmetric matrices, when $\mathrm{CC}$ gives only inequalities. As a preliminary step, this calculus should give complete continuity of the semi-group associated with the Cauchy problem for a multidimensional scalar conservation law.

Let us turn towards the Euler system for a barotropic gas:

$$
\begin{aligned}
\partial_{t} \rho+\operatorname{div}(\rho v) & =0, \\
\partial_{t}(\rho v)+\operatorname{Div}(\rho v \otimes v)+\nabla p(\rho) & =0 .
\end{aligned}
$$

Let us assume that $p^{\prime}>0$ (hyperbolicity) and either $(\rho p)^{\prime \prime}$ does not vanish (genuine non-linearity, or $p \equiv p_{\infty}-\frac{a^{2}}{\rho}$ (Chaplygin gas). When the initial data is $H^{s}$ with $s>1+\frac{d}{2}$, the standard theory of symmetric hyperbolic systems ensures that a unique solution exists, locally in time. Because of the singularity at the vacuum, one has to work in appropriate variables $(h(\rho), v)$, see $[2,21]$.

So far, the existence of global-in-time solutions is an open problem, unless either $d=1$ (as mentionned in Section 1), or the initial data is so special (see [11, 24]) that the solution remains classical for every $t>0$. In one space dimension, two sorts of techniques are available. That based on BV estimates (Glimm scheme, front tracking) is unlikely to extend in several space dimensions, because of the clues that $B V\left(\mathbb{R}^{d}\right)$ cannot be a space of well-posedness (see J. Rauch [23]), a phenomenon associated with the dispersiveness of the Euler system (and of most multi-D systems as well). The other technique is Compensated Compactness.

At first glance, Compensated Compactness is hopeless in dimension $d \geq 2$, for two reasons:

- In one space dimension, L. Tartar's strategy [29], developed by DiPerna [7] and followers involves infinitely many entropies. Such a large family always exists for $2 \times 2$ systems (two unknowns, two independent variables), but are unlikely in the other cases. This is the reason why CC has not been applied to the one-dimensional full gas dynamics, which consists in 3 equations. Even for an isentropic gas, the space of entropies is finite dimensional if $d \geq 2$. Thus we may deal only with finitely many independent entropy-flux pairs.

- For $2 \times 2$ systems, we have at our disposal the so-called div-curl Lemma, which is an equality. In higher space-time dimension (3 or more independent variables), the information provided by $\mathrm{CC}$ consists only in inequalities. Specifically, we expect that for some approximate solutions $u^{\epsilon}$ bounded in $L_{\text {loc }}^{2}$, some vector fields $\vec{q}^{\alpha}\left(u^{\epsilon}\right)$ are such that the sequence $\operatorname{div} \vec{q}^{\alpha}\left(u^{\epsilon}\right)$ remains 
in a compact subset of $H_{\mathrm{loc}}^{-1}\left(\mathbb{R}^{1+d}\right)$. Say that $\alpha=1, \ldots, r ;$ if $Q: \mathbf{M}_{r \times(1+d)}(\mathbb{R})$ is a quadratic form satisfying

$$
(\operatorname{det} M=0) \Longrightarrow(Q(M) \geq 0)
$$

then a Young measure associated with the sequence $\left(u^{\epsilon}\right)_{\epsilon>0}$ must satisfy

$$
\langle\nu, Q(M(u))\rangle \geq Q(\langle\nu, M(u)\rangle)
$$

where $u \mapsto M(u)$ is the matrix-valued function whose rows are the vector fields $\vec{q}^{\alpha}$. Equivalently, we have

$$
\langle\nu \otimes \nu, Q(M(v)-M(u))\rangle \geq 0,
$$

where the measure $\nu \otimes \nu$ acts on continuous functions of the doubled variable $(v, u) \in \mathbb{R}^{n} \times \mathbb{R}^{n}$.

The inequality (3) (or equivalently (4)) is quite poor in practice, and CC has never been applied to hyperbolic conservation laws in three or more spacetime dimensions. Even in the case of scalar conservation law $(n=1)$, only the one-dimensional (two space-time dimensions) has been treated by CC.

The direction that I suggest to investigate invokes another property of those systems of physical interest: the conservation laws to be solved have a symmetric form. This is clear in gas dynamics, because the Euler equations can be written as

$$
\operatorname{Div}_{t, x} S=0, \quad S=\left(\begin{array}{cc}
\rho & \rho v^{T} \\
\rho v & \rho v \otimes v+p I_{d}
\end{array}\right)
$$

Therefore the ambient space to consider in CC is not that of arbitrary matrices, but that of symmetric matrices $\mathbf{S y m}_{1+d}(\mathbb{R})$. My opinion is that we may even forget about the equation of energy, at least when applying Murat-Tartar's inequality (3). After all, it is already used to get an a priori estimate in $L_{t}^{\infty}\left(L^{2}\right)$, and its presence should break the symmetry.

Thus let us apply CC only to the sequence $S^{\epsilon}:=S\left(\rho^{\epsilon}, v^{\epsilon}\right)$. Even if we have only one such sequence, instead of an infinity, and only inequalities instead of an equality, we do have a lot of them. Namely, we obtain as many inequalities as there are quadratic form $Q: \mathbf{S y m}_{1+d} \rightarrow \mathbb{R}$ such that

$$
(\operatorname{det} S=0) \Longrightarrow(Q(S) \geq 0) \text {. }
$$

There are lot of such forms. For instance,

$$
Q_{0}(S)=d \operatorname{Tr}\left(S^{2}\right)-(\operatorname{Tr} S)^{2}
$$

works, because it rewrites in terms of the eigenvalues $\lambda_{0}, \ldots, \lambda_{d}$ of $S$ as

$$
d \sum_{j=0}^{d} \lambda_{j}^{2}-\left(\sum_{j=0}^{d} \lambda_{j}\right)^{2}
$$

which is non-negative when $\lambda \in \mathbb{R}^{1+d}$ has one zero component, by Cauchy-Schwarz inequality. From $Q_{0}$, we may also construct $Q_{P}: S \mapsto Q_{0}\left(P^{T} S P\right)$ whenever $P \in$ $\mathbf{M}_{1+d}(\mathbb{R})$. If $P$ is singular, then $Q_{P}$ is non-negative definite, and Murat-Tartar's 
inequality is just a consequence of Jensen's Inequality. On the contrary, (3) is nontrivial for $Q_{P}$ if $P$ is non-singular.

In practice, the set of quadratic forms suitable for $\mathrm{CC}$ is a convex cone, with a compact section. It is sufficient to write the Murat-Tartar inequality for each extremal point of this section. The first step would therefore to identify accurately this cone. It can be seen that this cone is strictly bigger than just that spanned by the $Q_{P}$ 's and the non-negative quadratic forms.

\section{Comments.}

- We do not expect to work out the CC for gas dynamics by following the above strategy, even in one space dimension. When $d=1$, all the $Q_{P}$ 's coincide and this amounts to apply the div-curl Lemma only once, a notoriously poor approach. Therefore we modestly suggest to push forward CC in a not-sohopeless situation, that of a multi-dimensional scalar conservation law.

- Our new approach is motivated by Tartar's calculus for the one-dimensional scalar equation

$$
\partial_{t} u+\partial_{x} f(u)=0, \quad u(t, x) \in \mathbb{R} .
$$

Tartar observed that it is enough to apply CC only once, to the entropy-flux pairs $(u, f)$ and $(f, \eta)$, where of course $\eta^{\prime}=f^{\prime 2}$. Then one uses the fact that for every $a, b \in \mathbb{R}$, we have

$$
(b-a)(\eta(b)-\eta(a)) \geq(f(b)-f(a))^{2},
$$

with equality only when $f$ is affine between $a$ and $b$. This choice of entropyflux pairs amounts to dealing with a symmetric object

$$
S=\left(\begin{array}{cc}
u & f(u) \\
f(u) & \eta(u)
\end{array}\right) .
$$

Actually, Tartar's calculation works out even in we invoke one inequality of the form (3), instead of the div-curl Lemma: it is enough to take $Q=$ - det. A natural generalization to a multi-dimensional scalar conservation law $\partial_{t} u+\operatorname{div} f(u)=0$ would be to work with the symmetric matrix $S(u)$ where

$$
s_{\alpha \beta}^{\prime}(u)=f_{\alpha}^{\prime}(u) f_{\beta}^{\prime}(u), \quad f_{0}(u) \equiv u .
$$

We point out that $S^{\prime}(u)$ is non-negative; incidentally, it is rank-one.

The good news are that in one space dimension, $S \mapsto-2 \operatorname{det} S$ is the special case of the $Q_{0}$ that we are allowed to deal with in several space dimensions.

\section{Compressible Navier-Stokes system with constant viscosities near vacuum.}

Problem \#3. In [13], D. Hoff and the author found an unphysical consequence of the hypothesis that the viscosity is a constant coefficient. This phenomenon occurs in a one space-dimension gas, in presence of vacuum. Is there a multi-dimensional counterpart?

Let us consider the isentropic or isothermal Navier-Stokes equations for a compressible fluid:

$$
\begin{aligned}
\partial_{t} \rho+\operatorname{div}(\rho v) & =0 \\
\partial_{t}(\rho v)+\operatorname{Div}(\rho v \otimes v)+\nabla p(\rho) & =\operatorname{Div} \mathcal{T},
\end{aligned}
$$


where the viscous tensor is given by

$$
\mathcal{T}:=\mu\left(\nabla v+(\nabla v)^{T}\right)+(\zeta-2 \mu)(\operatorname{div} v) I_{d}
$$

Let us assume that the viscosity coefficients are constant. This is not physically realistic, yet it is assumed in some mathematical works, either for convenience or because it is needed to work out the analysis. This assumption has the drawback that when the density vanishes, there remains a non-trivial equation for the velocity, namely

$$
\mu \Delta v+(\zeta-\mu) \nabla \operatorname{div} v=0 .
$$

This suggest that when considering a sequence of initial data $\left(\rho_{0}^{m}, u_{0}^{m}\right)$ with $\rho_{0}^{m}>0$, tending to a limit $\left(\bar{\rho}_{0}, \bar{u}_{0}\right)$ which has a zone of vaccum $\mathcal{V}_{0}$, then the limit $\bar{u}$ of the velocities $u^{m}(t, x)$ will satisfy $(5)$. This "ghost" velocity could have some effect onto the boundary of the vacuum zone $\mathcal{V}(t)$, like if there remained some gas in this domain, or like if the vacuum had its own pressure.

The scenario described above has been rigorously justified in one space dimension by D. Hoff and the author [13]. Specifically, the limit data $\left(\bar{\rho}_{0}, \bar{u}_{0}\right)$ was that of two droplets of gas separated by vacuum. As long as the drops don't merge, they evolve independently, with their own constant masses $m_{r, \ell}$ and momenta $q_{r, \ell}$. Their centers of mass move at constant speeds $v=q / m$. Assuming $v_{r}>v_{\ell}$, we deduce that the drops must merge at a time

$$
T^{*}<\frac{v_{\ell}-v_{r}}{L}
$$

with $L$ the distance between the centers of mass at initial time. On the contrary, when the initial data is modified by the addition of a small density $\epsilon>0$ between the drops, we observe that the drops remain separated by a wake of length $\geq R$, where $R$ does not depend upon $\epsilon$ and $t>0$. Therefore the limit of the corresponding solution is not the solution associated with the limit of the initial data: the solution does not depend continuously upon the initial data when the density tends to zero somewhere. Actually, the limit solution displays an unphysical transfer of momentum between both droplets.

The Question is whether this scenario remains valid in several space dimensions. We still may consider two droplets, such that the centers of mass and the velocities satisfy

$$
G_{\ell}-G_{r}+\tau\left(v_{\ell}-v_{r}\right)=0
$$

for some $\tau>0$. Then we know that the drops must merge at some time $T^{*}<\infty$ (mind that $T^{*}$ might be larger than $\tau$, as the center of mass of a droplet may not belong to the droplet itself, if it is not convex). Suppose now that the surrounding vacuum is replaced by an arbitrarily small density $\epsilon>0$. What can we say about the distance $r$ between the drops as a function of $\epsilon$ and $t$ ? Does it remain uniformly positive? This is not crystal-clear, because the added gas may escape in the directions tranversal to the axis between the drops. Or, is it possible that the merging of the drops is delayed, in the sense that there exists a time $T^{\prime}>T^{*}$ such that

$$
\liminf _{\epsilon \rightarrow 0} r\left(\epsilon, T^{\prime}\right)>0 ?
$$


A possibly simpler problem is that of an annulus, or of a spherical shell of gas, enclosing a zone of vacuum. Assume a rotational invariance of the data. Then the solution is radial:

$$
\rho=\rho(t, r), \quad v=v_{r}(t, r) \vec{e}_{r} .
$$

If $\rho_{0}^{\epsilon} \equiv \epsilon$ over some interval $\left(0, r_{-}\right)$, what happens when $\epsilon \rightarrow 0^{+}$? Let the vacuum zone at time $t$ be $(0, r(\epsilon, t))$. Does $r$ remain bounded away from zero, uniformly in $\epsilon$ and $t$ ? On the contrary, how does the vaccum zone $(0, r(t))$ behave when the data satisfies $\rho_{0} \equiv 0$ over $\left(0, r_{-}\right)$?

There has been quite a lot of work in the past decade, dealing with flows in presence of vacuum. See for instance [14] and the references therein. This paper suggests an interesting criterion (equation (1.9)) in order to select a unique solution when the density vanishes.

\section{Entire solutions of compressible Euler system.}

Problem \#4. Does there exist a non-trivial smooth entire (eternal) solution of the Euler system for compressible fluids, with finite mass and energy, in odd space-dimension?

In [24], we showed that the forward Cauchy problem for the Euler system of either an isentropic or a non-isentropic gas admits a global-in-time classical solution provided that the initial density is small enough, the initial entropy is close to a constant and the initial velocity is close to a linear field $\bar{u}(x)=A x$ where the spectrum of $A$ does not intersect $(-\infty, 0]$. As usual, the smallness and the closeness are understood in $H^{s}$ with $s>1+\frac{d}{2}$. M. Grassin [11] dropped the assumption that the velocity field is almost linear, by assuming only that at every point $x$, the Jacobian $\nabla_{x} u(0, x)$ does not have a non-positive eigenvalue, this in some uniform way. Let us point out that this global well-posedness was a new result for a non-isentropic gas even in one spacedimension. Only in the one-dimensional isentropic case this result met the litterature; see D.-Q. Li \& W.-C. Yu [18].

The data under consideration has typically finite mass and energy. For instance, the gas may occupy initially a bounded domain. Its support spreads as time increases, being bounded at every time. Of course, the diameter of the support grows unboundedly as $t \rightarrow+\infty$.

An amazing fact is that when the dimension $d$ is even (say 2, in order to be meaningful), then a $d \times d$ matrix may have no real eigenvalue at all. If this is the case for $\nabla_{x} u(0, x)$, then the above result applies both forward and backward (just change $(t, x) \leftrightarrow(-t,-x))$. For instance, if $u(0, x)$ is close to the field

$$
\bar{u}(x)=\left(\begin{array}{c}
-x_{2} \\
x_{1}
\end{array}\right),
$$

we obtain classical solutions that are defined for all $t \in \mathbb{R}$. Such solutions are called either entire (to mimic complex variable functions) or eternal.

On the contrary, the $2 \times 2$ case of a one-dimensional isentropic gas does not admit such a finite mass entire solution; this can be proved, following Lax, by differentiating twice the Riemann invariants along characteristic lines.

This suggest the following Question: Assume that $(\rho, u, s)$ is a classical solution of the Euler equation defined for all $t \in \mathbb{R}$ (and not only for $t>0$ ). We may consider 
either the isentropic gas with $p=\rho^{\gamma}$, or the non-isentropic with $p=(\gamma-1) \rho e$, with a constant $\gamma>1$. Assume in addition that the total mass and energy are finite and non-zero (for instance, the gas occupies at each time a bounded domain). Notice that this forbids the data to depend only upon $d-1$ coordinates. Is it true that the space dimension $d$ is even? Or is it possible to have such entire solutions in space dimension $3 ?$

Morally, if an entire solution exists, we anticipate the following scenario. The particles on the boundary of the domain are somehow those with largest velocity; they asymptotically behave as free particles. Let $\Phi_{s}^{t}$ be the flow map, which brings the particles from time $s$ to time $t$. We normalize the fluid domain so that it becomes the unit ball, hence being independent of the time $t$. We renormalize as well the flow map so that it belongs to $\operatorname{Diff}\left(B_{d}\right)$. For large $s<0<t$, the trace of renormalized flow map on the unit sphere is likely to belong to the connected component of $-\mathrm{id}_{d}$ within $\operatorname{Diff}\left(\mathbb{S}^{d-1}\right)$. If $d$ is odd, this contradicts the fact that the flow map preserves the orientation.

REMARK. If we drop the assumption that the solutions are smooth, there even exist solutions that are compactly supported in space and time, hence are eternal; see $[6]$.

\section{Regular reflection of a gas against a wedge.}

Problem \#5. Existence of a Regular Reflection for a compressible flow, without an irrotational assumption.

The problem of shock reflection along corners has a long history, begining with experiments done by E. Mach at the end of the XIXth century. From the mathematical point of view, one focuses on an ideal situation where the corner is the apex of a straight, infinite wedge, and the incident shock is planar, separating two uniform states. Of course, the state surrounding the wedge is at rest. The problem is to describe mathematically (existence, uniqueness, regularity, qualitative features) the flow after the time $t=0$ at which the shock hits the tip of the wedge.

For the sake of simplicity, we limit ourselves to the two-dimensional case and to barotropic fluids. The problem has finitely many data, say the aperture angle $2 \alpha$ of the wedge, two densities $\rho_{\text {down }}$ and $\rho_{\text {up }}$ (if we assume an isentropic flow) and the angle

$\beta$ of incidence of the shock. This determines the net mass flux $j$ per unit time across the shock, by

$$
j^{2}\left[\frac{1}{\rho}\right]+[p(\rho)]=0 .
$$

Then the normal shock velocity is given by $\rho_{0} V=j$. With $\beta$, this determines $\vec{V}$. Finally, the upstream velocity $v_{\text {up }}$ is given by $\rho_{1}\left(v_{\text {up }} \cdot N-V\right)=j, N$ being the (given) normal to the shock.

In practice, the mathematical analysis is restricted to the symmetric case where $\beta=0$; then the axis of symmetry, a line normal to the shock, which separates the sector into two equal parts, is equivalent to a rigid boundary.

The expected solution can be rather complicated in some cases. The simplest situation arises when either $\rho_{1}-\rho_{0}$ is small, or $\alpha$ is close enough to $\frac{\pi}{2}$. The reason is easy to understand: 


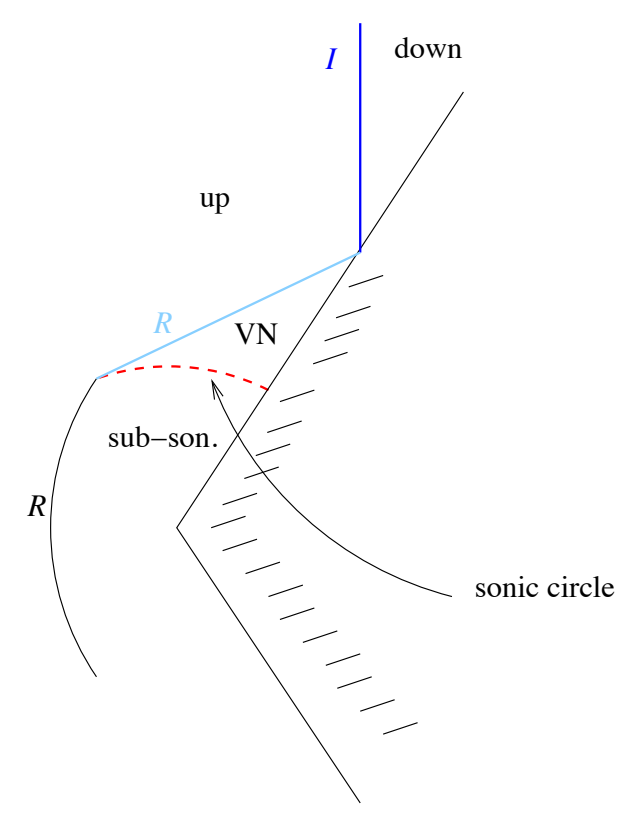

FIG. 1. The Regular Reflection. The solid wedge is at right. The reflected shock $R$ is straight until it meets $S$. Then it becomes a curve.

- If $\rho_{1}=\rho_{0}$, there is no shock at all, and the solution is just the rest state of density $\rho_{0}$,

- If instead $\alpha=\frac{\pi}{2}$, then the data (shock and domain) depend only upon the normal coordinate, and the reflexion reduces to a one-dimensional problem, which can be solved by algebraic calculations.

This "simple" situation is called a Regular Reflection (RR). Roughly speaking, the incident shock $I$ keeps moving, but reflects along the (rigid) wall of the sector. The reflected shock $R$ is at first a straight line, which separates the upstream state from another uniform state $U_{\mathrm{VN}}$ (after von Neumann). The latter is used to define a pseudo-sonic circle $S$, normal to the wall. When $R$ meets $S$, it becomes a curve and we lack an explicit description: mathematically speaking, the part of $R$ beyond $S$ is a free boundary. In the bounded domain delimited by $R, S$ and the wedge, the flow is (pseudo-)sub-sonic and non-constant; see Figure 1. Unlike the data, the solution cannot be described in finite terms. More, we don't know yet the existence of a solution to this problem.

We point out that the data are scale-invariant: both the domain (a wedge) and the state at $t=0$ are unchanged under $x \mapsto \lambda x$ when $\lambda>0$. Because the flow is governed by the Euler equations, a first-order system of conservation law, we expect a self-similar solution. This will certainly be true if the Cauchy problem has the uniqueness property. Self-similarity is that the unknown $(\rho, v)$ depends only upon $y:=\frac{x}{t}$. Then, introducing the pseudo-velocity $w:=v-y$, the Euler system reduces to the differential equations

$$
\begin{array}{r}
\operatorname{div}(\rho w)+2 \rho=0 \\
\rho(w \cdot \nabla) w+\rho w+\nabla p=0 .
\end{array}
$$


This is a first-order system of three equations in three unknowns, whose principal part is in conservation form. Its type depends upon the sign of $|w|-c(\rho)$, where $c=p^{\prime}$ is the sound speed. It is hyperbolic when $|w|>c$ (the pseudo-supersonic domain). The pseudo-sonic circle has therefore an equation $\left|y-v_{\mathrm{VN}}\right|=c\left(\rho_{\mathrm{VN}}\right)$.

Our Question is therefore to prove the existence of a solution of $(6,7)$, subject to the boundary conditions given by the RR data.

For more details, the reader is advised to have a look to the review paper [27] and to the the references herein.

Why is this problem difficult? Some difficulties of this problem can be overcome with modern analytical tools: - the fact that $R$ is a free boundary, - the type degeneracy along $S$ (see for instance [28]). Some others are significantly harder:

- In the subsonic domain, the system $(6,7)$ is neither hyperbolic nor elliptic, but something in between. Two characteristics are complex conjugate (a glimpse of ellipticity), but one remains real (a sign of hyperbolicity). It is impossible to decouple these opposite aspects. This is typically a situation where we lack ideas and tools.

- A calculation done in [27] shows that the vorticity $\omega=$ curl $v$ may not be square integrable! Thus not only the solution is not classical (because of the presence of shocks), but it is even not piecewise smooth. No satisfactory description of this vortical singularity has been given yet.

These fundamental difficulties led many people to simplify the problem by making the irrotational assumption. This is only an approximation of the reality, because the reflected shock, once it becomes curved, does generate non-zero vorticity. At first glance, this approximation seems relevant when the shock strength $\rho_{1}-\rho_{0}$ is very small, because the entropy generation is of the order of $\left(\rho_{1}-\rho_{0}\right)^{3}$; it is however definitely incompatible with the lack of integrability of $\omega^{2}$ in the real case.

The irrotational case displays two nice features. On the one hand, it prevents from the vortical singularity, and therefore we may expect a piecewise smooth solution. On the other hand, the system $(6,7)$ reduces to a single, second-order equation governing the stream function $\phi$ defined by $w=\nabla \phi$ :

$$
\operatorname{div}(\rho \nabla \phi)+2 \rho=0, \quad \rho=h^{-1}\left(-\phi-\frac{1}{2}|\nabla \phi|^{2}\right),
$$

where $\rho \mapsto h(\rho)$ is defined by $h^{\prime}(\rho)=\frac{1}{\rho} p^{\prime}(\rho)$. The situation is much better here than in the non-irrotational case: away from the sonic line, the equation is either hyperbolic or elliptic. Actually, it is elliptic in the domain where the solution is not piecewise constant. One may therefore use all the weapons of the scalar elliptic theory: maximum principle, Harnack inequality and so on. This is the strategy followed by G.-Q. Chen and M. Feldman [4], who ultimately proved the existence of an irrotational solution when $\rho_{1}-\rho_{0}$ is not too big and $\alpha$ is close enough to $\frac{\pi}{2}$.

Of course, it would be desirable to solve the non-irrotational problem instead. Pointwise a priori estimates for the density and its inverse, for the velocity and temperature (if the flow is non-isentropic) have been given in [27].

Another open question, related to this one, is whether the proof of the existence of a vortical singularity in the isentropic case can be extended to the non-isentropic case (full Euler equations).

Finally, let us mention that the three-dimensional situation, where the obstacle is a circular cone, is significantly more complicated. Even if we use the cylindrical 
symmetry of the problem, the reflected shock is curved immediately after it reflects along the wall. The state between $R$ and the wall is no longer uniform. Both $R$ and this state become unknowns. Of course, the sonic line is no longer a circle. To our knowledge, there have not been any mathematical paper on this more realistic problem, even under the irrotational assumption.

Concluding remarks. Even if one million dollars is awarded to a problem about viscous incompressible fluid dynamics, it is worth studying questions about compressible flows, with or without dissipative process. They may be more relevant in the applications. They present a greater variety of phenomena: the non-linearity is often competing with either dispersion or dissipation, in such a way that discontinuities can emerge, if the data is not too small. Such lack of smoothness raises a serious problem regarding the tools we should employ; standard functional spaces (Sobolev, Besov, $B V$, etc...) are not appropriate, at least in several space dimensions. Besides, compressibility allows the fluid to develop a cavity; this vaccum problem can be harmful to technical devices, as well as disturbing at the mathematical level, due to singularities in the equations or to type degeneracy. Last but not least, the coupling between hyperbolic and parabolic modes yields an interesting challenge in the choice of functional spaces, as well as in the choice of methods.

Compressible fluid dynamics is a very active field. The open problems are countless, and our choice of five of them is strongly biased by our own mathematical interest and previous research. There is some hope that they be solved in a way or another in the near future. We encourage young researchers to think about them, without any preconceived idea. It is often useful not to know too much about a field, in order to be able to develop truly new and efficient ideas.

\section{REFERENCES}

[1] D. Bresch And B. Desjardins, On the existence of global weak solutions to the Navier-Stokes equations for viscous compressible and heat conducting fluids, J. Math. Pures Appl., 87 (2007), pp. 57-90.

[2] J.-Y. Chemin, Dynamique des gaz à masse totale finie, Asymptotic Anal., 3 (1990), pp. 215220.

[3] M. Bae, G.-Q. Chen, And M. Feldman, Regularity of solutions to regular shock reflection for potential flow, Invent. math., 175 (2009), pp. 505-543.

[4] G.-Q. Chen and M. Feldman, Potential theory for shock reflection by a large-angle wedge, Proc. Natl. Acad. Sci. USA, 102 (2005), pp. 15368-15372. Global solutions of shock reflection by large-angle wedges for potential flow, Annals of Maths., 171 (2010), pp. 1067-1182.

[5] G.-Q. Chen and M. Perepelitsa, Vanishing viscosity limit of the Navier-Stokes equations to the Euler equations for compressible fluid flow, Comm. Pure Appl. Math., 63 (2010), pp. 1469-1504.

[6] C. De Lellis and L. SzÉKelyhidi, The h-principle and the equations of fluid dynamics, Bull. of the A. M. S., 49 (2012), pp. 347-375.

[7] R. DiPerna, Convergence of the viscosity method for isentropic gas dynamics, Comm. in Math. Physics, 91 (1983), pp. 1-30.

[8] X.-X. Ding, G.-Q. Chen, And P.-Z. Luo, Convergence of the fractional step Lax-Friedrichs scheme and Godunov scheme for the isentropic system of gas dynamics, Comm. Math. Phys., 121 (1989), pp. 63-84.

[9] E. FeIREISL, On the motion of a viscous, compressible, and heat conducting fluid, Indiana Univ. Math. J., 53 (2004), pp. 1705-1738.

[10] E. Feireisl, Dynamics of viscous compressible fluids, Oxford Univ. Press, Oxford (2004).

[11] M. Grassin, Global smooth solutions to Euler equations for a perfect gas, Indiana Univ. Math. J., 47 (1998), pp. 1397-1432.

[12] D. Hoff, Discontinuous solutions of the Navier-Stokes Equations for multidimensional flows of heat-conducting fluids, Arch. Rat. Mech. Anal., 139 (1997), pp. 303-354. 
[13] D. Hoff AND D. SERRE, The failure of continuous dependence on initial data for the NavierStokes equations of compressible flow, SIAM J. Appl. Math., 51 (1991), pp. 887-898.

[14] X. Huang, J. LI, AND Z. XIN, Global well-posedness of classical solutions with large oscillations and vacuum to the three-dimensional isentropic compressible Navier-Stokes equations, Comm. Pure Appl. Math., 65 (2012), pp. 549-585.

[15] S. KAWASHIMA, Systems of a hyperbolic parabolic type with applications to the equations of magnetohydrodynamics, PhD Thesis, Kyoto University (1983).

[16] S. KaWAShima AND Y. Shizuta, On the normal form of the symmetric hyperbolic-parabolic systems associated with the conservation laws, Tóhoku Math. J., 40 (1988), pp. 449-464.

[17] Ph. LeFloch, Entropies and weak solutions of the compressible isentropic Euler equations, C.R.A.S., series I 324 (1997), pp. 1105-1110.

[18] D.-Q. Li AND W.-C. YU, Solutions régulières globales des systèmes hyperboliques quasi linéaires d'ordre 1, J. Maths. Pures Appl., 4 (1982), pp. 401-409.

[19] P.-L. Lions, B. Perthame, And P. Souganidis, Existence and stability of entropy solutions for the hyperbolic systems of isentropic gas dynamics in Eulerian and Lagrangian coordinates, Comm. Pure and Appl. Math., 49 (1996), pp. 599-638.

[20] P.-L. Lions, B. Perthame, and E. Tadmor, Kinetic formulation of the isentropic gas dynamics and p-systems, Comm. Math. Phys., 163 (1994), pp. 415-431.

[21] T. Makino, S. UkaI, And S. Kawashima, Sur la solution à support compact de l'équation d'Euler compressible, Japan J. Appl. Math., 3 (1986), pp. 249-257.

[22] T. NishidA, Global solution for an initial boundary value problem of a quasilinear hyperbolic system, Proc. Japan Acad., 44 (1968), pp. 642-646.

[23] J. RAUCH, BV estimates fail for most quasilinear hyperbolic systems in dimension greater than one, Comm. in Math. Physics, 106 (1986), pp. 481-484.

[24] D. SERRE, Solutions classiques globales des équations d'Euler pour un fluide parfait compressible, Ann. Inst. Fourier, 47 (1997), pp. 139-153.

[25] D. SERRE, Viscous system of conservation laws: Singular limits, Nonlinear Conservation Laws and Appl., A. Bressan, G.-Q. Chen, M. Lewicka, D. Wang eds. IMA volume in Maths \& Appl., 153, Springer-Verlag (2010), pp. 433-446.

[26] D. SERRE, The structure of dissipative viscous system of conservation laws, Physica, D293 (2010), pp. 1381-1386.

[27] D. SERRE, Shock reflection in gas dynamics, Handbook of Mathematical Fluid Dynamics, vol. IV. Eds: S. Friedlander, D. Serre. Elsevier, North-Holland (2007), pp. 39-122.

[28] D. Serre And H. FreistüHLer, The hyperbolic/elliptic transition in the multi-dimensional Riemann Problem, Indiana Univ. Math. J., (2012).

[29] L. TARTAR, Compensated compactness and applications to partial differential equations, Nonlinear analysis and mechanics: Heriot-Watt Symposium, Vol. IV, Res. Notes in Math., 39, Pitman, Boston-London, 1979, pp.136-212. 
D. SERRE 